\title{
Genetically modified mouse models to help fight COVID-19
}

\author{
Channabasavaiah B. Gurumurthy $\oplus^{1,2 \bowtie}$, Rolen M. Quadros ${ }^{2}$, Guy P. Richardson ${ }^{3}$, \\ Larisa Y. Poluektova $\oplus^{1}$, Suzanne L. Mansour ${ }^{4}$ and Masato Ohtsuka $\oplus^{5,6 凶}$
}

The research community is in a race to understand the molecular mechanisms of severe acute respiratory syndrome coronavirus 2 (SARS-CoV-2) infection, to repurpose currently available antiviral drugs and to develop new therapies and vaccines against coronavirus disease 2019 (COVID-19). One major challenge in achieving these goals is the paucity of suitable preclinical animal models. Mice constitute $\sim 70 \%$ of all the laboratory animal species used in biomedical research. Unfortunately, SARS-CoV-2 infects mice only if they have been genetically modified to express human ACE2. The inherent resistance of wild-type mice to SARS-CoV-2, combined with a wealth of genetic tools that are available only for modifying mice, offers a unique opportunity to create a versatile set of genetically engineered mouse models useful for COVID-19 research. We propose three broad categories of these models and more than two dozen designs that may be useful for SARS-CoV-2 research and for fighting COVID-19.

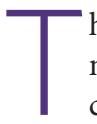
he research community is in desperate need of animal models for testing therapeutics and vaccines against coronavirus disease 2019 (COVID-19), and for studies aimed at understanding the molecular mechanisms of severe acute respiratory syndrome (SARS) coronavirus (CoV) 2 (SARS-CoV-2) infection and pathogenesis. A wide spectrum of laboratory and domestic animal species have been challenged with SARS-CoV-2 in an effort to find suitable models, but only a few, such as hamsters, cats, ferrets and monkeys, are susceptible $^{1-3}$. Unfortunately, many of these species are neither accessible nor practical for the vast majority of researchers to make rapid progress.

Mice are the most commonly used laboratory animals in biomedical research, and they have helped scientists develop diagnostics, therapeutics and vaccines for many human diseases. As such, it would have been easy to use mice for COVID-19 research if they were susceptible to SARS-CoV-2 infection, like humans, but that is not the case. One way to render mice susceptible to SARS-CoV-2 is to genetically modify them.

Evaluating the utility of GEMMs previously developed for SARS-CoV research

Several genetically engineered mouse model (GEMMs) that express the hACE2 gene were created over a decade ago for use in SARS virus studies ${ }^{4-7}$. Like SARS-CoV, SARS-CoV-2 uses the hACE2 receptor for entry into cells. Therefore some of these transgenic models have been investigated for their suitability for SARS-CoV-2 studies ${ }^{8,9}$. Repurposing of previously made GEMMs and non-GEMMs (discussed in the next section), including animals other than mice, for the study of novel coronaviruses has been extensively reviewed by Yuan et al. ${ }^{10}$ and Singh et al. ${ }^{11}$. Even though the existing hACE2 transgenic mice fully support SARS-CoV-2 replication, the pathogenesis does not accurately model the disease course seen in humans ${ }^{12}$, and the models also have other limitations. First, because these models still express the mouse orthologue of ACE2 (mACE2), this will occupy a portion of the cell surface pool of total ACE2, leading to non-physiological amounts of the human ACE2 available for virus interactions. Second, the transgenic expression cassettes in current models are driven by different promoters, and often expressed in different types of cells and at levels that are not similar to endogenous mACE2 expression. Furthermore, constitutively expressing transgenic mouse models are not suited to experiments requiring spatiotemporal regulation of hACE2 expression (i.e., in specific cell types, such as lung epithelial cells, and/or at specific time points). Finally, these models will not be ideal for researchers wanting to simulate comorbid conditions such as obesity, hypertension and diabetes, in which affected tissues will have altered ACE2 expression $^{13,14}$.

${ }^{1}$ Department of Pharmacology and Experimental Neuroscience, College of Medicine, University of Nebraska Medical Center, Omaha, NE, USA. ${ }^{2}$ Mouse Genome Engineering Core Facility, Department of Pharmacology and Experimental Neuroscience, College of Medicine, University of Nebraska Medical Center, Omaha, NE, USA. ${ }^{3}$ Sussex Neuroscience, University of Sussex, Falmer, Brighton, UK. ${ }^{4}$ Department of Human Genetics, University of Utah, Salt Lake City, UT, USA. ${ }^{5}$ Division of Basic Medical Science and Molecular Medicine, Department of Molecular Life Science, School

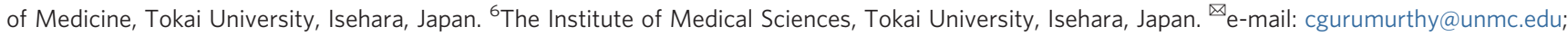
masato@is.icc.u-tokai.ac.jp 


\section{Mouse models developed by using non-GEMM approaches}

Because the scientific community has such an urgent need for preclinical models, some groups explored non-GEMM strategies that were also previously used for SARS-CoV studies, such as (i) adapting the virus by serial passage through mice so that infectious mutant strains emerge ${ }^{15}$, and (ii) delivering hACE2encoding DNA through adenovirus (AdV) vector systems followed by infection of the mice with SARS-CoV. Both of these strategies were demonstrated recently as proof of principle for SARS-CoV-2 ${ }^{12,16-18}$. Adaptation of SARS-CoV-2 virus to mice ${ }^{4}$ did not cause lethality, but the approach seems useful for testing vaccine efficacy ${ }^{12,16}$. Delivering hACE2 into mice through an $\mathrm{AdV}$ vector system demonstrated the utility of the approach for pathogenicity studies, testing vaccines and therapeutics ${ }^{17,18}$. Some limitations of the AdV vector approach, however, include the requirement for large quantities of vector and mouse-to-mouse variability in the response to infection. As most mice do not develop severe disease ${ }^{19}$, they do not develop the extrapulmonary manifestations seen in humans ${ }^{17}$, and some animals show lung pathology resulting from AdV administration itself $^{18}$. Direct comparisons of GEMMs versus non-GEMMs show that GEMMs provide a stable system for testing the ability of vaccines and antivirals to protect against disease, whereas non-GEMMs offer the flexibility to be used across multiple genetic backgrounds ${ }^{19}$.

\section{Versatility of GEMMs}

Although the previously generated transgenic hACE2 mice and the non-GEMM approaches have successfully established that mice can be re-tooled as preclinical models for COVID-19 studies, sophisticated GEMM approaches provide a much more versatile suite of COVID-19 models than can be envisioned for any other species. The natural non-permissiveness of mice to SARS-CoV-2 infection combined with the wide variety of available genetic tools and molecular switches offers a unique opportunity to make this species even more useful for COVID-19 research.

Researchers are now facing a familiar COVID-19 narrative: trying to make sense of a mystifying illness ${ }^{20}$ affecting not only the respiratory system, but also many other organs, including those of the cardiovascular ${ }^{21}$, digestive ${ }^{22}$ and nervous systems $^{23}$. If a researcher wants to study the pathophysiological and molecular events occurring only in the heart, intestine or brain, it would be difficult to study such events in an animal model in which many other organs are also infected (e.g., lung or kidney). If the animal model allowed productive infection of only one type of tissue or cell (e.g., cardiac, endothelial or neuronal), and if the model allowed regulation of the dose and timing of infection, it would be very helpful in answering a variety of research questions relevant to different types of tissue. Related to this, a recent report described the expression pattern of ACE2 in human tissues. Although somewhat intriguing, expression of ACE2 seemed to be very low in lung cells, whereas it was detected at high levels in $>150$ cell types corresponding to 45 tissues $^{24}$. This observation further highlights the value of the conditional and inducible expression models where researchers working in a wide range of tissue types can use the models to ask specific questions by limiting $\mathrm{hACE} 2$ expression and viral infection to only a subset of tissue types.

This type of controlled experimental design is possible only in mice because the genetic tools and molecular switches (e.g., Cre-LoxP and tetracycline-inducible systems) are readily available for multiple cell types and tissue systems. For example, a CRE-activatable hACE2 knockin mouse model, once created, could be bred to any of the thousands of already available Cre driver lines, thus allowing researchers from a range of biomedical fields, from cardiac physiologists to neuroscientists, to use this model for probing their particular research questions of interest. In addition, individual gene knockout (KO) mouse models are available for thousands of genes. The molecular pathways of viral infection can be further dissected through classical mouse genetic studies by breeding the COVID-19 models with a number of different gene KO models. Thus, mice can be very useful models in the fight against COVID-19. In this Perspective, we propose concepts for developing more than two dozen GEMMs suitable for various research studies on COVID-19 and SARS-CoV-2.

We propose the following three broad categories of GEMMs for COVID-19 research: (i) knocking-in expression cassettes, or point mutations, into the endogenous mouse Ace2 locus; (ii) knocking-in CRE-activatable- or tetracycline-inducible-hACE2 expression cassettes into safe-harbor loci, by re-engineering the existing reporter or inducer lines; and (iii) knocking-in CREactivatable cassettes into the mouse Ace 2 locus. The proposed GEMMs are listed in Table 1; the schematics of the GEMM designs are shown in Figs. 1-3.

GEMM category 1: knocking-in expression cassettes or point mutations into the endogenous mouse Ace 2 locus One of the ideal GEMMs for COVID-19 research is a knockout:knockin (KO:KI) design in which the mouse Ace 2 gene is deleted (KO), and the human ACE2 cDNA is inserted in its place, termed 'model no. 1' herein. Both mouse and human ACE2 genes are located on the $\mathrm{X}$ chromosome, and each contains 19 exons. Although all corresponding exons are the same size, the mouse introns are larger, with a total gene size of 49 and $41 \mathrm{~kb}$ in mice and humans, respectively. The KO:KI model can be generated by deleting the region between the start and stop codons of mouse Ace2 (i.e., all coding exons and the introns between them) and inserting the 2.4-kb hACE2 cDNA. In the KO:KI design, because $\mathrm{mAce} 2$ is deleted, any potential confounding effects on the response to virus infection will not occur, unlike in the hACE2 transgenic mice with random insertions. In this model, the mouse Ace 2 promoter is expected to drive human ACE2 cDNA expression at physiological levels, although the effect of deleting the mouse introns cannot be ascertained, as they may contain regulatory regions. While our manuscript was under preparation, a model similar to this design was reported in which the hACE2 cDNA was inserted near the start codon of the mouse $A c e 2^{25}$. Although the $\mathrm{mAce} 2$ genomic region is still retained in this model, $\mathrm{mAce} 2$ is not likely to be expressed, because the insertion 


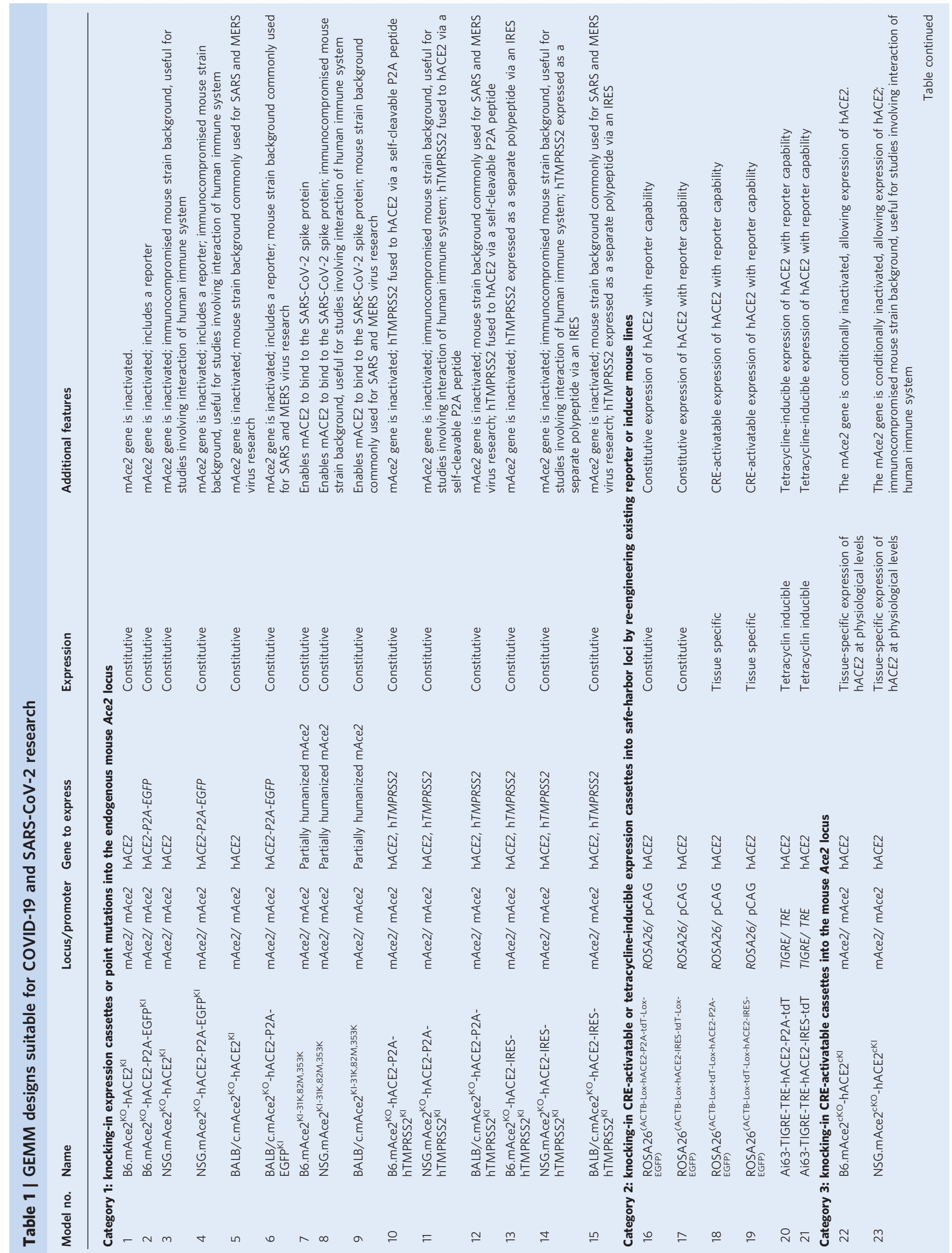




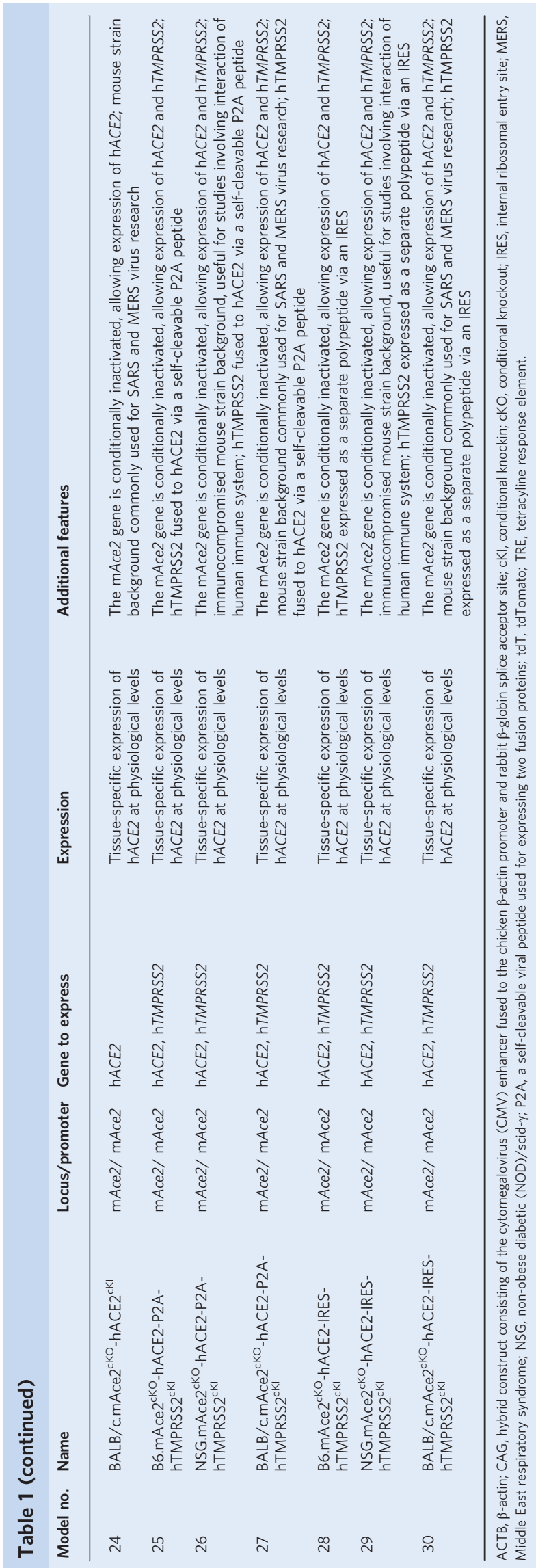

cassette contains a poly (A) sequence upstream of the $\mathrm{mAce} 2$ coding exons. This design also includes additional regulatory elements such as the woodchuck hepatitis virus posttranscriptional regulatory element, which enhances mRNA stability and translation efficiency and may produce higherthan-physiological hACE2 protein levels. The ideal model design would be one that can achieve near-physiological levels of hACE2 expression. Such a model design would require precise replacement of all of the 19 mouse exons with the corresponding human exons, leaving the mouse introns, which may contain regulatory sequences, intact. Developing such a model would, however, be quite difficult. One other feature included in the published model ${ }^{25}$ is a fluorescent reporter (tdTomato (tdT)) expressed as a separate protein via an internal ribosomal entry site (IRES) element, with the reporter serving as a handy tool to mark the hACE2-expressing cells. We propose including a self-cleaving viral peptide $\mathrm{P} 2 \mathrm{~A}$, instead of the IRES element, fused to the $\mathrm{C}$ terminus of hACE2, followed by the EGFP coding sequence in model no. 2 .

10-15 y ago, generating a KO:KI model like the one described above would have required multiple lengthy steps of complex gene targeting in embryonic stem cells, single-cell cloning and chimera breeding. The process would typically take over 1 y just to develop a founder mouse, and this may be the reason why such a model was not created. In addition, it is possible that when SARS-CoV research declined, scientists were not motivated to invest the necessary time and resources. Now, the situation is quite different; the COVID-19 pandemic has increased the demand for any GEMM available for SARSCoV-2 infection, even though the existing models are suboptimal for certain COVID-19 research designs. On the bright side, GEMM technologies have improved tremendously during the last decade because of the advent of CRISPR technology ${ }^{26-35}$. Thus, more sophisticated GEMMs (e.g., the KO:KI model) can be created within the time span of a few months.

In addition, CRISPR tools offer many more technical options than were previously possible ${ }^{36}$. For example, GEMMs can be created in any strain background. Although we propose that models no. 1 and no. 2 be made in the C57BL/6 reference mouse strain, which is immunocompetent, we also propose that these design strategies are implemented in an immunocompromised mouse strain background, such as non-obese diabetic (NOD)/scid- $\gamma$ (NSG) ${ }_{c}{ }^{\text {null }}$ (NSG/NOG). NSG mice lack mature T cells, B cells and natural killer cells ${ }^{37}$. These GEMMs (no. 3 and no. 4) will be useful in many ways. First, because of the immunosuppressed background, there may be uncontrolled SARS-CoV-2 replication, which would make this strain particularly suitable for screening antiviral drugs. Second, the NSG/NOG strain is very commonly used for transplantation of human hematopoietic stem cells, which leads to the development of a humanized immune system ${ }^{38-41}$. These models will be suitable for pathogenesis studies involving interactions of the human immune system with the virus and can lead to better understanding of the role of human macrophages permissive for SARS-CoV-2 and mechanisms of human immunopathogenesis ${ }^{42}$. Third, some COVID-19 patients exhibit cytokine storm syndromes and immunosuppression ${ }^{43}$, which are the result of active viral replication in an immune-deficient 
$\mathbf{a}$
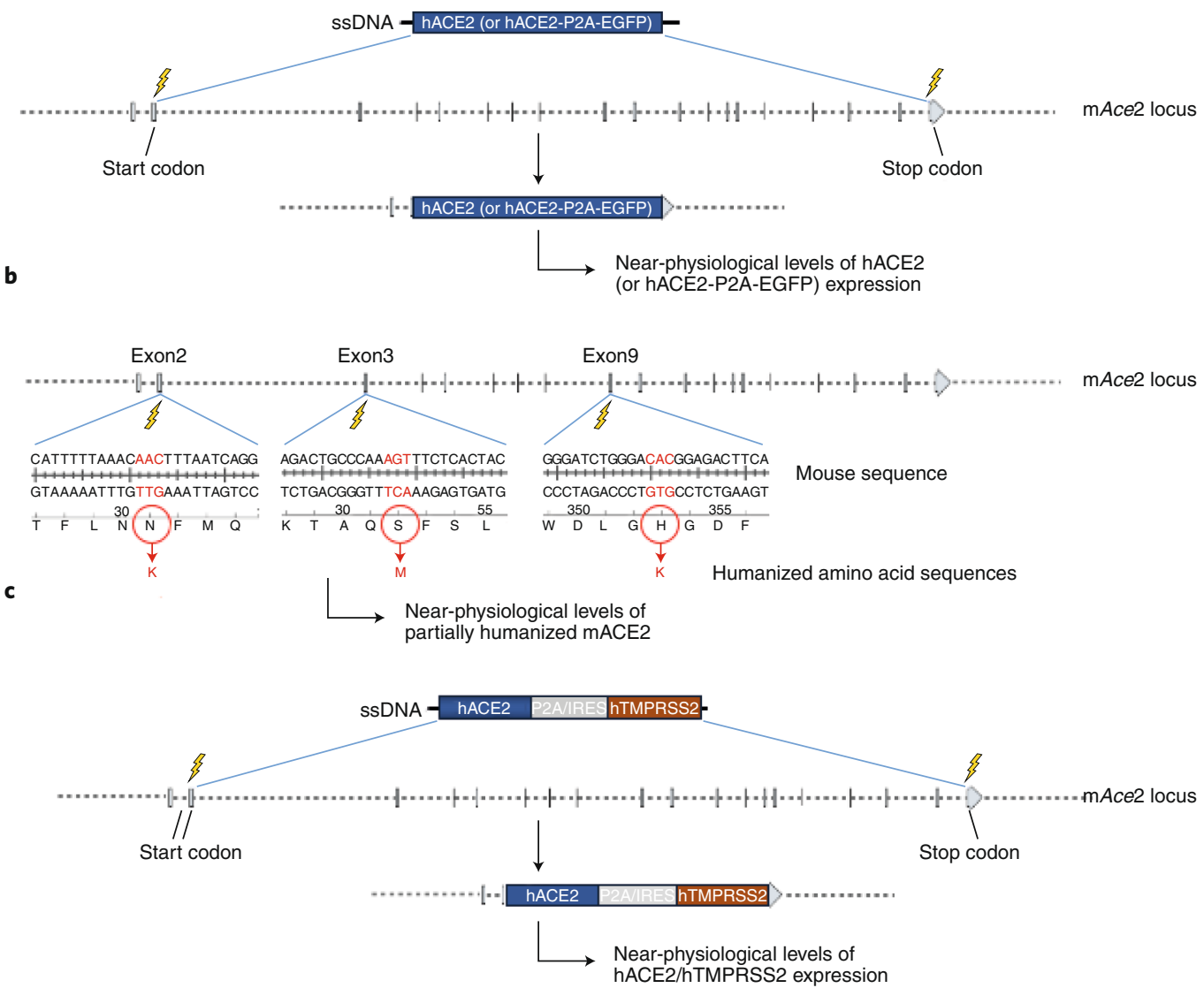

Fig. 1 | Schematics of COVID-19 GEMM designs, category 1: knocking-in expression cassettes or point mutation changes into the endogenous mouse Ace2 locus. a, KO:KI design; human ACE2 cDNA (GEMMs no. 1, no. 3 and no. 5) or human ACE2 cDNA fused to P2A-EGFP GEMMs no. 2, no. 4 and no. 6) will be inserted in place of mouse Ace2 by deleting $48 \mathrm{~kb}$ of genomic sequence between the start and stop codons of $\mathrm{mAce} 2$. $\mathbf{b}, \mathrm{Key}$ amino acids KI design (GEMMs no. 7-9); only the key amino acids that differ between mice and humans and are responsible for binding to the spike protein will be replaced by knocking-in a single-stranded oligodeoxynucleotide (ssODN) donor containing the humanized codons. The locations of amino acids N31K, S82M and H353K are shown. c, KO:KI designs to co-express hACE2 and hTMPRSS2 (GEMMs no. 10-15). IRES, internal ribosomal entry site; P2A, a self-cleavable viral peptide used for expressing two fusion proteins.

host. Therefore, GEMMs allowing SARS-CoV-2 infection in immunocompromised backgrounds can serve as valuable tools for such immunological studies. Last, humanization of NSG/ NOG mice with hACE2 will provide the ability to study the reaction of human immune cells in viral replication. This can be achieved by creating additional genetic modifications in GEMMs, which will allow more sophisticated immunological studies involving transplanting and infecting human cells into mice, similar to studies with $\mathrm{HIV}^{44,45}$. Considering that the $\mathrm{BALB} / \mathrm{c}$ is one of the most commonly used mouse strains used for SARS and Middle East respiratory syndrome (MERS) virus research $^{46,47}$, we also propose developing the KO:KI model in the BALB/c background strain (models no. 5 and no. 6). For more information on additional strain considerations, see Future directions.

As an alternative to the KO:KI design, one could mutate the amino acids of mACE2 into those required for hACE2 binding to the SARS-CoV-2 spike protein. Crystal structures ${ }^{48,49}$ showed that five residues (K31, E35, D38, M82 and K353) are critical for receptor binding ${ }^{50}$. Of these, residues K31, M82 and
K353 differ in mice. Therefore, changing just these three residues could allow mACE2 to bind effectively to the SARSCoV-2 spike protein and permit infection of expressing cells. This strategy would retain any regulatory elements within the mouse introns, and it should result in nearly physiological levels of a functional mouse ACE2 receptor. These models are termed no. 7 , no. 8 and no. 9 in C57BL/6, NSG/NOG and $\mathrm{BALB} / \mathrm{c}$ strains, respectively. A similar point-mutation KI strategy in which two amino acids (288 and 300) of the DPP4 receptor for MERS-CoV were mutated to render mice susceptible to MERS-CoV was reported previously ${ }^{51}$.

Transmembrane protease, serine 2 (TMPRSS2) is a protease important in cleaving hACE2 and SARS-CoV-2 spike proteins, enabling efficient viral entry into cells ${ }^{52}$. It is possible $\mathrm{h} A C E 2$ expression alone in mice will be insufficient to permit infection and viral pathogenesis at a level similar to humans. However, co-expression of hTMPRSS2 may achieve this level. Models no. 10-15 (and no. 25-30: see below in category 3) are proposed for the co-expression of hTMPRSS2. KI designs for the purpose of this co-expression would involve either fusing TMPRSS2 to 


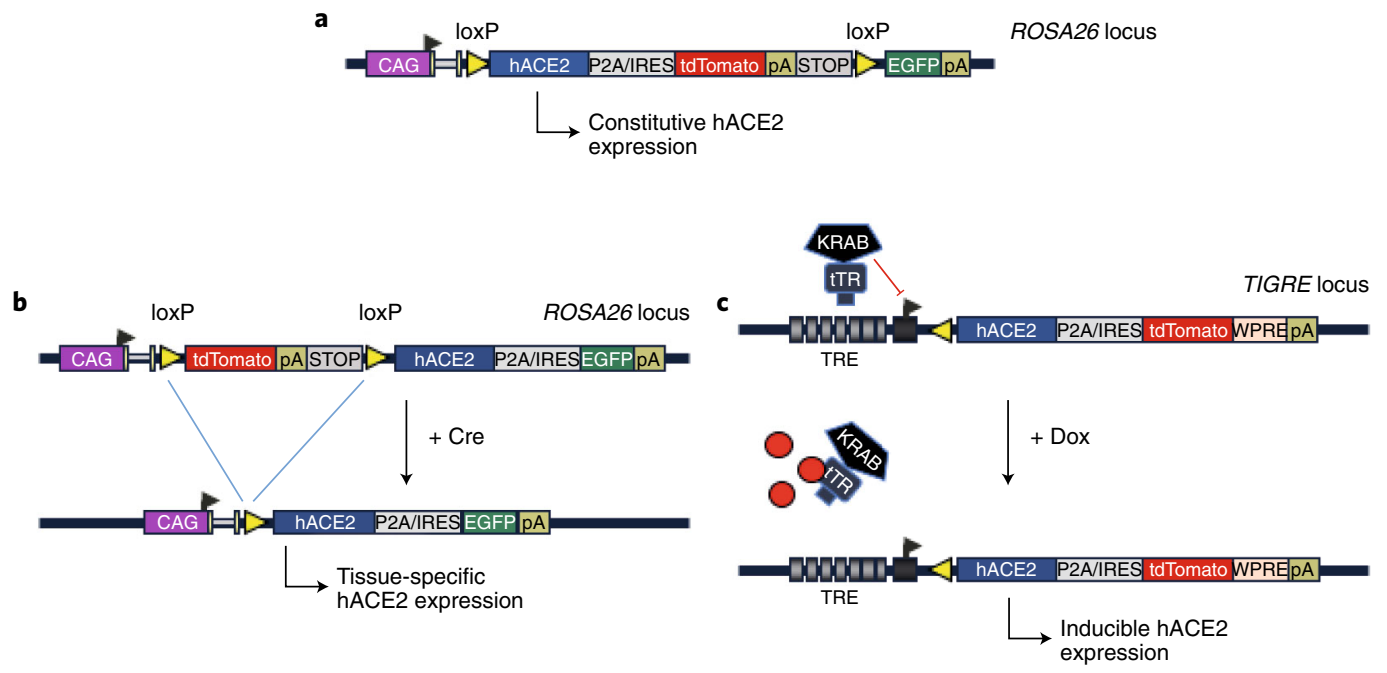

Fig. 2 | Schematics of COVID-19 GEMM designs, category 2: knocking-in CRE-activatable or tetracycline-inducible expression cassettes into safe-harbor loci by re-engineering existing reporter or inducer mouse lines. a-c, Constitutive (a), CRE-activatable (b) and tetracycline-inducible (c) designs (GEMMs no. 16-21). These models can be generated by re-engineering existing Cre reporter and tetracycline-inducible models. CAG, hybrid construct consisting of the CMV enhancer fused to the chicken $\beta$-actin promoter and rabbit $\beta$-globin splice acceptor site; IRES, internal ribosomal entry site; KRAB, Krüppel-associated box; P2A, a self-cleavable viral peptide used for expressing two fusion proteins; TRE, tetracycline response element; tTR, tetracycline repressor; WPRE, woodchuck hepatitis virus post-transcriptional regulatory element.

the $\mathrm{C}$ terminus of the hACE2 KI cassette via a self-cleavable $\mathrm{P} 2 \mathrm{~A}$ peptide, or expressing TMPRSS2 as a separate polypeptide via an IRES. These designs are similar to models no. 1 , no. 3 and no. 5 but contain P2A-hTMPRSS2 for C-terminus fusion (models no. 10-12) or IRES elements (models no. 13-15). In certain cases, fusion of additional peptide sequences (e.g., P2A) may affect protein folding and function, which can be avoided by using IRES elements. However, the gene downstream of the IRES element may not express as efficiently as the gene placed upstream of it. A strategy of co-expressing hACE2 and hTMPRSS2, similar to the ones we proposed here, was also proposed by Soldatov et al. ${ }^{53}$, although these authors suggested inserting the expression cassettes into the mTMPRSS2 locus instead of the mAce 2 locus. Co-expression of hTMPRSS2 may not be necessary in the case of the key amino acid KI mutant models (no. 7-9) because the mACE2 will probably retain its endogenous cleavage site for mouse TMPRSS2 proteins. It is unknown, however, whether mTMPRSS2 will cleave SARSCoV-2 spike proteins.

\section{GEMM category 2: knocking-in CRE-activatable or tetracycline-inducible expression cassettes into safe- harbor loci by re-engineering existing reporter or inducer mouse lines}

Models no. 1-15 will express hACE2 alone or in conjunction with hTMPRSS 2 in all cells in which the $\mathrm{mAce} 2$ promoter is active. There will, however, be many situations in which scientists require GEMMs for answering very specific questions. For example, what molecular events are perturbed when only cardiac, intestinal or lung epithelial cells in the mouse are infected with SARS-CoV-2? Such a scenario would require a GEMM expressing hACE2 in only one of the respective cell types, and this expression could be switched on or off when needed.

Although models no. 1-15 do not offer flexibility to control the timing or tissue specificity of expression, there are sophisticated molecular genetic switches, such as Cre-LoxP and tetracycline-inducible systems, that can be included in GEMM designs to allow such defined experimental scenarios. By leveraging the vast numbers of available Cre driver lines created by the mouse genetics community, CRE-activatable KI mice can be bred to those driver lines to remove the stop cassette and to express the insertion cassette. For example, a KI mouse containing a LoxP-Stop-LoxP cassette between the promoter and hACE2 cassette will express hACE2 only after the removal of the stop cassette, and this can be achieved by breeding the KI mouse with a Cre driver line. Similarly, a KI mouse in which a tetracycline response element drives $\mathrm{hACE} 2$ expression could be used for turning on or off expression of $\mathrm{hACE} 2$ in any desired cell type at any given time point. Numerous tetracycline-inducible models that express the tetracycline transactivator (tTA) or reverse tTA have been generated by the community and can be bred with the KI model to turn on or off hACE2 expression simply by administering/ withdrawing doxycycline. The conditional and inducible expression systems described below will also offer a degree of biosafety compared to constitutively expressing models, as they will likely not produce potentially infectious virus particles before the start of the experiments.

GEMMs no. 16 and no. 17 offer constitutive expression with a reporter capability, and models no. 18 and no. 19 offer CREactivatable features. These models will contain hACE2 fused to a fluorescent reporter, such as EGFP or tdT, either through a self-cleavable $\mathrm{P} 2 \mathrm{~A}$ peptide or expressed as a separate protein via an IRES. The four models will be useful for many different 


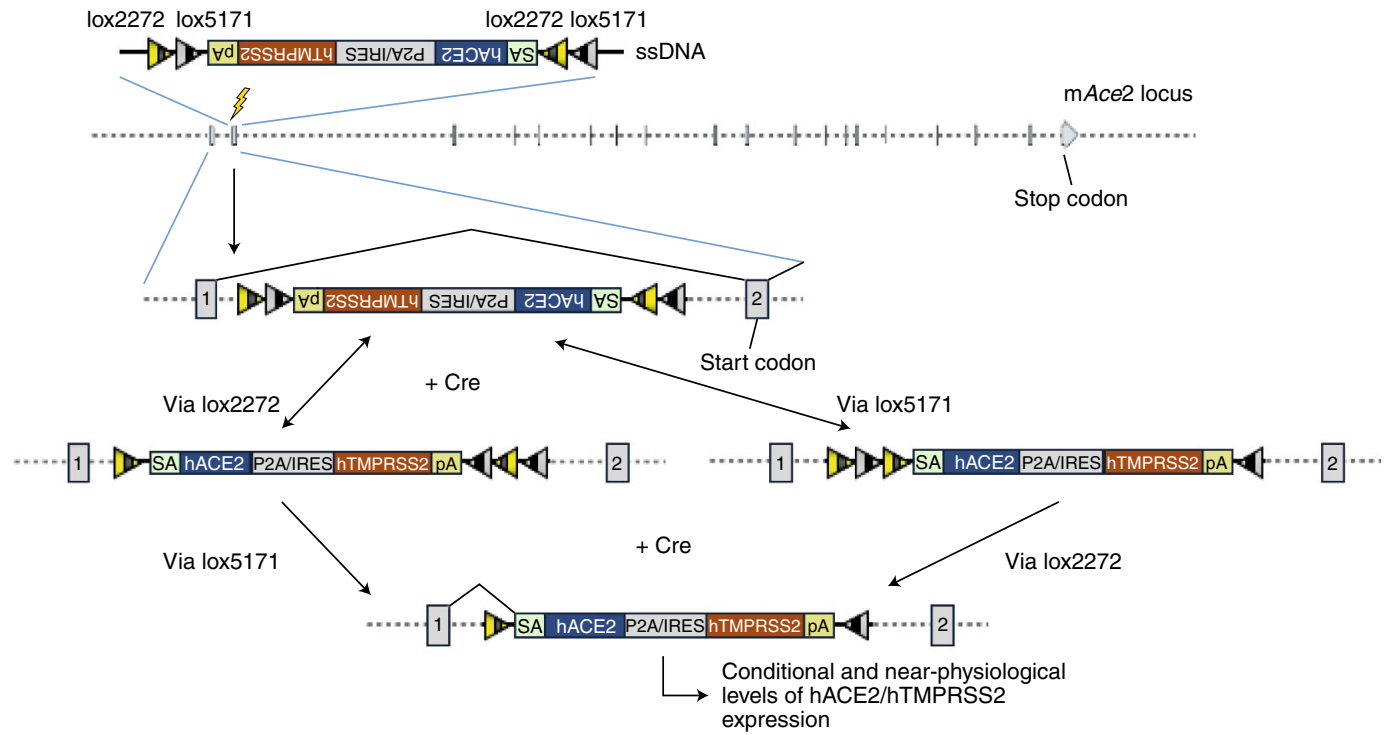

Fig. 3 | Schematics of COVID-19 GEMM designs, category 3: knocking-in CRE-activatable cassettes into the mouse Ace2 locus. A CRE-invertible KI cassette is inserted in the opposite orientation to the $\mathrm{mAce} 2$ locus. Upon CRE-mediated inversion of the cassette through sequential recombinations of lox2272s and lox5171s, the transcript is spliced from exon1 to the inverted KI cassette, and downstream transcription is terminated to prevent mAce2 function. This CRE-mediated inversion allows hACE2 expression in models no. 22-24 (not shown) or hACE2 and hTMPRRS2 expression in models no. 25-30. IRES, internal ribosomal entry site; P2A, a self-cleavable viral peptide used for expressing two fusion proteins; SA, splice acceptor; ssDNA, single-stranded DNA.

scenarios, including constitutive expression, tissue-specific expression and turning on or off expression of the receptor as needed, simply by breeding them with Cre driver lines (in the case of models no. 18 and no. 19), which are available for thousands of promoters. Fluorescent reporters in animal models will offer a useful tool for histological and biochemical experiments. Models no. 20 and no. 21 offer tetracyclineinducible features wherein the expression cassettes are placed downstream of tetracycline response elements. As above, these models will contain hACE2 fused to a fluorescent reporter either through a self-cleavable $\mathrm{P} 2 \mathrm{~A}$ peptide or expressed as a separate protein via IRES. The expression of the transgenes can be achieved in an inducible fashion by breeding the models with tTA/reverse tTA driver lines and through doxycycline administration.

These KI models (no. 16-21) can be developed by reengineering the genomes of some of the previously developed GEMMs, by using CRISPR approaches. This re-engineering approach will allow the use of available GEMMs harboring proven genetic elements such as $\operatorname{LoxP}$, tetracycline response elements, polyA signals and built-in reporters. For generating models no. 16-19, for example, a reporter mouse model called ' $\mathrm{mT} / \mathrm{mG}$ ' offers a suitable model for re-engineering. The $\mathrm{mT} /$ $\mathrm{mG}$ double-fluorescent Cre reporter mouse contains a floxed cassette of membrane-targeted $\operatorname{tdT}(\mathrm{mT})$ followed by a membrane-targeted green fluorescent protein $(\mathrm{mG})$, targeted into the ROSA26 locus, one of the most commonly used safe harbor sites in the mouse genome. The $\mathrm{mT}$ reporter is expressed in every mouse cell. Once the Cre recombinase is introduced via breeding, the $\mathrm{mT}$ cassette is deleted, allowing the CAG (CMV enhancer fused to the chicken $\beta$-actin promoter and rabbit $\beta$-globin splice acceptor site) promoter to express the $\mathrm{mG}$ reporter ${ }^{54}$. This mouse strain is available for purchase from The Jackson Laboratory (JAX stock no. 007676). To create tetracycline-inducible KI models (no. 20 and no. 21), the mouse strain Ai63 can be re-engineered to insert the hACE2 cDNA near the start codon of the tdT sequence, either as a fusion via a $\mathrm{P} 2 \mathrm{~A}$ peptide or with an IRES. The Ai63 mouse was developed in Hongkui Zeng's laboratory at the Allen Brain Research Institute in Seattle and expresses $\mathrm{tdT}$ under the tetracycline response element (TRE) promoter ${ }^{55}$.

\section{GEMM category 3: knocking-in CRE-activatable cassettes into the mouse Ace 2 locus}

Another advance in developing models for COVID-19 would be to combine the features of knocking-in expression cassettes with conditional potential. In models no. 22-24, CREactivatable inversion cassettes expressing hACE2, flanked by a combination of mutant LoxP elements (Lox2272 and 5171) ${ }^{56}$ are inserted into intron 1 of mouse Ace2. The coding sequences are placed in the opposite orientation to the $\mathrm{mAce} 2$ promoter. Upon CRE-mediated recombination, the coding sequences will be inverted, thereby placing them in the correct orientation to be expressed from the $m A c e 2$ promoter, enabling the promoter to drive expression of $\mathrm{hACE} 2$. In models no. 25-30, the inversion cassettes express both hACE2 and hTMPRSS2 (either fused to P2A or as separate proteins using an IRES between them). We call this design, used in models no. 22-30, a conditional KI (cKI) strategy. These GEMMs are expected to express near-physiological levels of hACE2 along with the required amounts of hTMPRSS2 upon CRE-mediated recombination. These models will be particularly suitable for research projects that address well-defined questions requiring selective 
expression of the two key proteins (hACE2 and hTMPRSS2) only in certain cell types of the mouse at a specific time point of interest. Although numerous Cre driver lines developed in the C57BL/6 strain (or the ones backcrossed to this strain) are readily available, such strains are not available for the NSG/ NOG and BALB/c strains. Considering that Cre driver lines can be generated by using robust technologies like EasiCRISPR $^{30,32}$ quite easily, such models can be generated as needed very rapidly ( 2 months' time to generating and identifying the G0 founder mice $)^{32}$.

Details of all 30 GEMM designs are given in Table 1. The full sequences of the targeting constructs and the guide RNA sequences, and additional notes on the models are given in Supplementary Note 1. The targeting constructs for these models, except the ones containing hTMPRSS2 coding sequences, are approximately $\leq 3 \mathrm{~kb}$ in length. These models can be generated by using the Easi-CRISPR approach, which uses long single-stranded DNA as a donor for highly efficient insertion of sequences into the mouse genome $\mathrm{e}^{30,32}$. The models containing hTMPRSS 2 sequences are longer than $4 \mathrm{~kb}$ and could also be generated by using the Easi-CRISPR approach, but the efficiency may be lower and require the injection of more zygotes to achieve the faithful insertion of a full-length cassette. Alternative strategies of using plasmid DNA constructs (circular double-stranded DNA (dsDNA)) containing much longer homology arms $\mathrm{s}^{25,28,57,58}$ or single-stranded oligodeoxynucleotides (ssODN)-mediated KI of large cassettes ${ }^{31}$ can be attempted in the event that the Easi-CRISPR method is not successful in generating the desired models containing hTMPRSS2.

\section{Concluding remarks}

Mice are the most commonly used laboratory animals in biomedical research, and they have helped scientists develop diagnostics, therapeutics and vaccines for many human diseases. Although the previously generated transgenic hACE2 mice and non-GEMM approaches (such as adaptation of virus to mice and adenovirus-mediated delivery of $\mathrm{hACE2}$ ) have been successfully validated as potential preclinical models for COVID-19 studies, more sophisticated GEMM approaches will render mice much more versatile COVID-19 models than can be envisioned for any other species. The availability of a wide variety of genetic tools and molecular switches available only in mice offers a unique opportunity to make this species useful for COVID-19 research. We described three broad categories of GEMMs and $\sim 30$ different model designs in three important mouse strains that may be useful for SARS-CoV-2 research and for fighting COVID-19. By using robust CRISPR-based strategies such as Easi-CRISPR ${ }^{30,32}$ and/or improved genomeediting via oviductal nucleic acid delivery $(i \text {-GONAD })^{33,34}$, the founder mice for most of these models, particularly those involving only insertion of $\mathrm{hACE} 2$ and point mutation KIs, can be generated within $\sim 2$ months. The designs involving both hACE2 and hTMPRSS2 insertion are more complex because of the longer insertion sizes. They may require the injection of larger numbers of zygotes to create KI models because as the size of the insertion cassettes become longer, the efficiency of full-length cassette insertion becomes lower. Nevertheless, even the simpler models will rapidly provide the opportunity for significant advances. Furthermore, the use of GEMM mice together with SARS-CoV-2 mouse-adapted virus ${ }^{12,16}$ may provide the ideal mouse model for COVID-19.

\section{Future directions}

COVID-19 is a new disease. There are many unknowns concerning SARS-CoV-2, including its pathogenicity. Nevertheless, the research community is making advances at a remarkable speed. At this stage, the proposed GEMMs suitable for COVID-19 and SARS-CoV-2 research are based on the available information. Additional models could include expressing soluble hACE2 ${ }^{59}$ along with FURIN protease (paired basic amino acid cleaving enzyme) ${ }^{60}$, with the latter cleaving and producing mature proteins required for viral entry. A recent study identified polymorphisms in the human ACE2 and TMPRSS2 genes that may explain variability in susceptibility to COVID- $19^{61}$. These polymorphisms could be engineered into mice for comparison with the proposed GEMMs expressing the standard human genes.

In addition, over the last century, the laboratory mouse has been bred to produce an extensive catalogue of inbred, hybrid and outbred strains, which collectively provide an elegant system suitable for studying many human disease conditions. This wealth of strain resources could be leveraged for SARSCoV-2 disease research. For example, the GEMM designs proposed here can be generated in additional mouse strains, taking into consideration SARS-CoV-2 co-morbidities and human genetic diversity. The New Zealand obese mouse strain could be useful for simulating SARS-CoV-2 co-morbidities ${ }^{62}$ of obesity and diabetes because of its natural insulin resistance and susceptibility to developing these conditions ${ }^{63}$. Hybrids of the New Zealand black and New Zealand white strains could be considered for simulating autoimmune conditions, as they have been used as models of human systemic lupus erythematosus ${ }^{64}$. Several inbred and hybrid strains have been tested for their suitability for performing studies of cardiac function $^{65}$, vascular properties ${ }^{66}$, hypertension ${ }^{67}$ and arrythmia ${ }^{68}$. These demonstrate a wide range of variability in assay performance between genetic backgrounds, however, suggesting that careful considerations should be made in choosing strain backgrounds for a given assay in cardiovascular disease research. Experimentation with the proposed GEMMs generated in different strains will show whether genetic backgrounds affect susceptibility to SARS-CoV-2 infection generally or in specific tissues. This may provide information on the genetic basis for the variability of COVID-19 symptoms in humans.

During the last decade, a novel set of strains called Collaborative Cross (CC) strains were also added to the vast array of mouse genetic backgrounds. CC strains are specially designed to overcome the narrow genetic diversity of inbred strains by crossing many inbred mouse lines together ${ }^{69}$. The complex mix of human genetic diversity is expected to be reflected in the mouse CC strains. These strains have been used for a wide range of studies, including identifying genetic modifiers affecting susceptibility for the pathogenicity of infectious 
diseases affecting human lungs ${ }^{70}$. Genome-wide studies have been performed by infecting CC strains with mouse-adapted SARS-CoV to identify polymorphic host genes that contribute to pathogenesis, and these have identified genes such as $\operatorname{Trim}_{55^{71}}$ and Ticam $2^{72}$. Related to this, human genome-wide association studies have identified genomic regions that may be important for SARS-CoV-2 susceptibility and severity. Interestingly, preliminary studies have identified a region of chromosome 3 p21.31 comprising six genes ${ }^{73,74}$, one of which is SLC6A20, a gene that encodes a transporter interacting with hACE2.

Incorporating factors like FURIN protease or polymorphic versions of ACE2, TMPRSS2 or SLC6A20 similar to our proposed GEMM designs, or producing our proposed GEMMs in additional strain backgrounds including CC strains can extend the COVID-19 GEMM list from no. 31 onward and vastly increase opportunities to understand COVID-19. Furthermore, using the mouse as the model system allows us to leverage the results of research into over three dozen genes that have already been investigated with respect to coronavirus disease (see Fig. 2 of a recent review by LoPresti et al. ) $^{75}$. Systematically breeding the 30 GEM models described here with each of the KOs for those three dozen genes will serve as a valuable tool to understand more about coronaviruses.

The list of 30 GEMMs proposed herein offers a foundation for the research community to generate the first set of small animal models for COVID-19 and SARS-CoV-2 research. These are expected to prove valuable preclinical models for evaluating COVID-19 therapeutics and vaccines, which may continue to be important for studying the next coronavirus that may jump from animals to people.

\section{References}

1. Shi, J. et al. Susceptibility of ferrets, cats, dogs, and other domesticated animals to SARS-coronavirus 2. Science 368, 1016-1020 (2020).

2. Rockx, B. et al. Comparative pathogenesis of COVID-19, MERS, and SARS in a nonhuman primate model. Science 368 , 1012-1015 (2020).

3. Sia, S. F. et al. Pathogenesis and transmission of SARS-CoV-2 in golden hamsters. Nature 583, 834-838 (2020).

4. Yang, X.-H. et al. Mice transgenic for human angiotensinconverting enzyme 2 provide a model for SARS coronavirus infection. Comp. Med. 57, 450-459 (2007).

5. McCray, P. B. et al. Lethal infection of K18-hACE2 mice infected with severe acute respiratory syndrome coronavirus. J. Virol. 81, 813-821 (2007).

6. Tseng, C.-T. K. et al. Severe acute respiratory syndrome coronavirus infection of mice transgenic for the human angiotensin-converting enzyme 2 virus receptor. J. Virol. 81, 1162-1173 (2007).

7. Menachery, V. D. et al. SARS-like WIV1-CoV poised for human emergence. Proc. Natl Acad. Sci. USA 113, 3048-3053 (2016).

8. Bao, L. et al. The pathogenicity of SARS-CoV-2 in hACE2 transgenic mice. Nature 583, 830-833 (2020).

9. Jiang, R.-D. et al. Pathogenesis of SARS-CoV-2 in transgenic mice expressing human angiotensin-converting enzyme 2. Cell 182, 50-58.e8 (2020).

10. Yuan, L., Tang, Q., Cheng, T. \& Xia, N. Animal models for emerging coronavirus: progress and new insights. Emerg. Microbes Infect. 9, 949-961 (2020).
11. Singh, A. et al. A comprehensive review of animal models for coronaviruses: SARS-CoV-2, SARS-CoV, and MERS-CoV. Virol. Sin. 35, 290-304 (2020).

12. Dinnon, K. H. et al. A mouse-adapted SARS-CoV-2 model for the evaluation of COVID-19 medical countermeasures. Preprint at http://biorxiv.org/lookup/doi/10.1101/2020.05.06.081497 (2020).

13. Winkler, E. S. et al. SARS-CoV-2 infection of human ACE2transgenic mice causes severe lung inflammation and impaired function. Nat. Immunol. 21, 1327-1335 (2020).

14. Li, Y., Zhou, W., Yang, L. \& You, R. Physiological and pathological regulation of ACE2, the SARS-CoV-2 receptor. Pharmacol. Res. 157, 104833 (2020).

15. Roberts, A. et al. A mouse-adapted SARS-coronavirus causes disease and mortality in BALB/c mice. PLoS Pathog. 3, e5 (2007).

16. $\mathrm{Gu}, \mathrm{H}$. et al. Adaptation of SARS-CoV-2 in BALB/c mice for testing vaccine efficacy. Science 369, 1603-1607 (2020).

17. Sun, J. et al. Generation of a broadly useful model for COVID-19 pathogenesis, vaccination, and treatment. Cell 182, 734-743.e5 (2020)

18. Hassan, A. O. et al. A SARS-CoV-2 infection model in mice demonstrates protection by neutralizing antibodies. Cell 182, 744-753.e4 (2020).

19. Rathnasinghe, R. et al. Comparison of transgenic and adenovirus hACE2 mouse models for SARS-CoV-2 infection. Preprint at https://www.biorxiv.org/content/10.1101/2020.07.06. 190066v1 (2020).

20. Couzin-Frankel, J. From 'brain fog' to heart damage, COVID-19's lingering problems alarm scientists. Science https://www. sciencemag.org/news/2020/07/brain-fog-heart-damage-covid-19s-lingering-problems-alarm-scientists (2020).

21. Lindner, D. et al. Association of cardiac infection with SARSCoV-2 in confirmed COVID-19 autopsy cases. JAMA Cardiol. e203551 (2020).

22. Parasa, S. et al. Prevalence of gastrointestinal symptoms and fecal viral shedding in patients with coronavirus disease 2019: a systematic review and meta-analysis. JAMA Netw. Open 3, e2011335 (2020).

23. Koralnik, I. J. \& Tyler, K. L. Covid -19: a global threat to the nervous system. Ann. Neurol. 88, 1-11 (2020).

24. Hikmet, F. et al. The protein expression profile of ACE2 in human tissues. Mol. Syst. Biol. 16, e9610 (2020).

25. Sun, S.-H. et al. A mouse model of SARS-CoV-2 infection and pathogenesis. Cell Host Microbe 28, 124-133.e4 (2020).

26. Shen, B. et al. Generation of gene-modified mice via Cas9/RNAmediated gene targeting. Cell Res. 23, 720-723 (2013).

27. Harms, D. W. et al. Mouse genome editing using the CRISPR/Cas system. Curr. Protoc. Hum. Genet. 83, 15.7.1-27 (2014).

28. Aida, T. et al. Cloning-free CRISPR/Cas system facilitates functional cassette knock-in in mice. Genome Biol. 16, 87 (2015).

29. Miura, H. CRISPR/Cas9-based generation of knockdown mice by intronic insertion of artificial microRNA using longer singlestranded DNA. Sci. Rep. 5, 12799 (2015).

30. Quadros, R. M. et al. Easi-CRISPR: a robust method for one-step generation of mice carrying conditional and insertion alleles using long ssDNA donors and CRISPR ribonucleoproteins. Genome Biol. 18, 92 (2017).

31. Yoshimi, K. ssODN-mediated knock-in with CRISPR-Cas for large genomic regions in zygotes. Nat. Commun. 7, 10431 (2016).

32. Miura, H., Quadros, R. M., Gurumurthy, C. B. \& Ohtsuka, M. Easi-CRISPR for creating knock-in and conditional knockout mouse models using long ssDNA donors. Nat. Protoc. 13, 195-215 (2018).

33. Ohtsuka, M. et al. $i$-GONAD: a robust method for in situ germline genome engineering using CRISPR nucleases. Genome Biol. 19, 25 (2018). 
34. Gurumurthy, C. B. et al. Creation of CRISPR-based germlinegenome-engineered mice without ex vivo handling of zygotes by i-GONAD. Nat. Protoc. 14, 2452-2482 (2019).

35. Gurumurthy, C. B. \& Lloyd, K. C. K. Generating mouse models for biomedical research: technological advances. Dis. Model. Mech. 12, dmm029462 (2019).

36. Gurumurthy, C. B. et al. CRISPR/Cas9 and the paradigm shift in mouse genome manipulation technologies. in Genome Editing (ed. Turksen, K.) 65-77 (Springer, 2016).

37. Shultz, L. D. et al. Human lymphoid and myeloid cell development in NOD/LtSz-scid IL2R $\gamma$ null mice engrafted with mobilized human hemopoietic stem cells. J. Immunol. 174, 6477-6489 (2005).

38. Ito, M. et al. NOD/SCID/gamma(c)(null) mouse: an excellent recipient mouse model for engraftment of human cells. Blood 100, 3175-3182 (2002).

39. Saito, Y. et al. The in vivo development of human $\mathrm{T}$ cells from $\mathrm{CD} 34^{+}$cells in the murine thymic environment. Int. Immunol. 14, 1113-1124 (2002).

40. Ishikawa, F. et al. Development of functional human blood and immune systems in NOD/SCID/IL2 receptor $\gamma$ chain $^{\text {null }}$ mice. Blood 106, 1565-1573 (2005).

41. Gorantla, S., Poluektova, L. \& Gendelman, H. E. Rodent models for HIV-associated neurocognitive disorders. Trends Neurosci. 35, 197-208 (2012).

42. Merad, M. \& Martin, J. C. Pathological inflammation in patients with COVID-19: a key role for monocytes and macrophages. Nat. Rev. Immunol. 20, 355-362 (2020).

43. Mehta, P. et al. COVID-19: consider cytokine storm syndromes and immunosuppression. Lancet 395, 1033-1034 (2020).

44. Dagur, R. S. et al. Human-like NSG mouse glycoproteins sialylation pattern changes the phenotype of human lymphocytes and sensitivity to HIV-1 infection. BMC Immunol. 20, 2 (2019).

45. Dash, P. K. et al. Sequential LASER ART and CRISPR treatments eliminate HIV-1 in a subset of infected humanized mice. Nat. Commun. 10, 2753 (2019).

46. Roberts, A. et al. Aged BALB/c mice as a model for increased severity of severe acute respiratory syndrome in elderly humans. $J$. Virol. 79, 5833-5838 (2005).

47. Chen, J. et al. Cellular immune responses to severe acute respiratory syndrome coronavirus (SARS-CoV) infection in senescent $\mathrm{BALB} / \mathrm{c}$ mice: $\mathrm{CD} 4^{+} \mathrm{T}$ cells are important in control of SARS-CoV infection. J. Virol. 84, 1289-1301 (2010).

48. Li, F., Li, W., Farzan, M. \& Harrison, S. C. Structure of SARS coronavirus spike receptor-binding domain complexed with receptor. Science 309, 1864-1868 (2005).

49. Wan, Y., Shang, J., Graham, R., Baric, R. S. \& Li, F. Receptor recognition by the novel coronavirus from Wuhan: an analysis based on decade-long structural studies of SARS coronavirus. J. Virol. 94, e00127-20 (2020).

50. Luan, J., Lu, Y., Jin, X. \& Zhang, L. Spike protein recognition of mammalian ACE2 predicts the host range and an optimized ACE2 for SARS-CoV-2 infection. Biochem. Biophys. Res. Commun. 526, 165-169 (2020).

51. Leist, S. R. \& Cockrell, A. S. Genetically engineering a susceptible mouse model for MERS-CoV-induced acute respiratory distress syndrome. in MERS Coronavirus: Methods and Protocols (ed. Vijay, R.) 137-159 (Springer, 2020).

52. Hoffmann, M. et al. SARS-CoV-2 cell entry depends on ACE2 and TMPRSS2 and is blocked by a clinically proven protease inhibitor. Cell 181, 271-280.e8 (2020).

53. Soldatov, V. O., Kubekina, M. V., Silaeva, Y. Yu., Bruter, A. V. \& Deykin, A. V. On the way from SARS-CoV-sensitive mice to murine COVID-19 model. Res. Results Pharmacol. 6, $1-7(2020)$
54. Muzumdar, M. D., Tasic, B., Miyamichi, K., Li, L. \& Luo, L. A global double-fluorescent Cre reporter mouse. Genesis 45, 593-605 (2007).

55. Daigle, T. L. et al. A suite of transgenic driver and reporter mouse lines with enhanced brain-cell-type targeting and functionality. Cell 174, 465-480.e22 (2018).

56. Robles-Oteiza, C. et al. Recombinase-based conditional and reversible gene regulation via XTR alleles. Nat. Commun. 6, 8783 (2015).

57. Abe, T., Inoue, K., Furuta, Y. \& Kiyonari, H. Pronuclear microinjection during S-phase increases the efficiency of CRISPR-Cas9assisted knockin of large DNA donors in mouse zygotes. Cell Rep. 31, 107653 (2020).

58. Yoshimi, K. et al. Combi-CRISPR: combination of NHEJ and HDR provides efficient and precise plasmid-based knock-ins in mice and rats. Hum. Genet. https://doi.org/10.1007/s00439-02002198-4 (2020).

59. Monteil, V. et al. Inhibition of SARS-CoV-2 infections in engineered human tissues using clinical-grade soluble human ACE2. Cell 181, 905-913.e7 (2020).

60. Andersen, K. G., Rambaut, A., Lipkin, W. I., Holmes, E. C. \& Garry, R. F. The proximal origin of SARS-CoV-2. Nat. Med. 26, 450-452 (2020).

61. Hou, Y. et al. New insights into genetic susceptibility of COVID19: an ACE2 and TMPRSS2 polymorphism analysis. BMC Med. 18, 216 (2020).

62. Richardson, S. et al. Presenting characteristics, comorbidities, and outcomes among 5700 patients hospitalized with COVID-19 in the New York City area. JAMA 323, 2052 (2020).

63. Veroni, M. C., Proietto, J. \& Larkins, R. G. Evolution of insulin resistance in New Zealand obese mice. Diabetes 40, 1480-1487 (1991).

64. Vyse, T. J. et al. Genetic linkage of IgG autoantibody production in relation to lupus nephritis in New Zealand hybrid mice. J. Clin. Invest. 98, 1762-1772 (1996).

65. Barnabei, M. S., Palpant, N. J. \& Metzger, J. M. Influence of genetic background on ex vivo and in vivo cardiac function in several commonly used inbred mouse strains. Physiol. Genomics 42A, 103-113 (2010).

66. Steppan, J. et al. Commonly used mouse strains have distinct vascular properties. Hypertens. Res. 43, 1175-1181 (2020).

67. Zhao, W. et al. Differential expression of hypertensive phenotypes in BXD mouse strains in response to angiotensin II. Am. J. Hypertens. 31, 108-114 (2018).

68. Jelinek, M., Wallach, C., Ehmke, H. \& Schwoerer, A. P. Genetic background dominates the susceptibility to ventricular arrhythmias in a murine model of $\beta$-adrenergic stimulation. Sci. Rep. 8, $2312(2018)$.

69. Threadgill, D. W., Miller, D. R., Churchill, G. A. \& de Villena, F. P.-M. The Collaborative Cross: a recombinant inbred mouse population for the systems genetic era. ILAR J. 52, 24-31 (2011).

70. Lorè, N. I. et al. Collaborative Cross mice yield genetic modifiers for Pseudomonas aeruginosa infection in human lung disease. mBio 11, e00097-20 (2020).

71. Gralinski, L. E. et al. Genome wide identification of SARS-CoV susceptibility loci using the Collaborative Cross. PLoS Genet. 11, e1005504 (2015).

72. Gralinski, L. E. et al. Allelic variation in the toll-like receptor adaptor protein Ticam 2 contributes to SARS-coronavirus pathogenesis in mice. G3 (Bethesda) 7, 1653-1663 (2017).

73. Ellinghaus, D. et al. Genomewide association study of severe Covid-19 with respiratory failure. N. Engl. J. Med. 383, 1522-1534 (2020).

74. The COVID-19 Host Genetics Initiative. The COVID-19 Host Genetics Initiative, a global initiative to elucidate the role of host genetic factors in susceptibility and severity of the SARS-CoV-2 virus pandemic. Eur. J. Hum. Genet. 28, 715-718 (2020). 
75. LoPresti, M., Beck, D. B., Duggal, P., Cummings, D. A. T. \& Solomon, B. D. The role of host genetic factors in coronavirus susceptibility: review of animal and systematic review of human literature. Am. J. Hum. Genet. 107, 381-402 (2020).

\section{Acknowledgements}

We thank H. Zeng, B. Tasic and T. Daigle (Allen Institute for Brain Science) for advice and discussions on the design of re-engineered alleles. We thank M. A. Montgomery and D. D. Meigs (University of Nebraska Medical Center) for professional editing. C.B.G. is funded by NIH grants R35HG010719, R21GM129559, R21AI143394 and R21DA046831. S.L.M. is funded by NIH grants R01DC011819 and R01DC014470. M.O. is funded by JSPS (16KK0189) for the Promotion of Joint International Research (Fostering Joint International Research).

\section{Author contributions}

C.B.G. and M.O. conceived the overall idea and wrote the initial manuscript. R.M.Q., G.P.R., L.Y.P. and S.L.M. contributed additional conceptual ideas and revised the manuscript. C.B.G., R.M.Q. and M.O. developed the model designs (supplementary file information).

\section{Competing interests}

The University of Nebraska Medical Center has filed a provisional patent on the proposed mouse model designs (inventors: C.B.G., R.M.Q. and M.O.).

\section{Additional information}

Supplementary information is available for this paper at https://doi.org/10.1038/ s41596-020-00403-2.

Correspondence and requests for materials should be addressed to C.B.G. or M.O. Peer review information Nature Protocols thanks Kun Li and the other, anonymous, reviewer(s) for their contribution to the peer review of this work.

Reprints and permissions information is available at www.nature.com/reprints.

Publisher's note Springer Nature remains neutral with regard to jurisdictional claims in published maps and institutional affiliations.

Received: 26 June 2020; Accepted: 9 September 2020;

Published online: 26 October 2020 